\title{
Medical complications of lung transplantation
}

\author{
R.M. Kotloff, V.N. Ahya
}

\begin{abstract}
Medical complications of lung transplantation. R.M. Kotloff, V.N. Ahya. (C)ERS Journals Ltd 2004.

ABSTRACT: Surgical advances, in conjunction with more effective immunosuppressive strategies, have propelled the field of lung transplantation forward and have made intermediate-term survival an achievable goal. Nonetheless, the post-transplant course is often marked by complications that threaten both the quality and duration of the recipient's life.

Many of the medical complications that arise are the direct consequence of the need to administer potent immunosuppressive agents, with their attendant risks of infection, malignancy and drug toxicity.

This article will review the major medical complications, excluding allograft rejection, which may be encountered in the lung transplant recipient. Familiarity with, and vigilance for, these problems should facilitate earlier recognition, more expeditious intervention and more favourable outcomes.

Eur Respir J 2004; 23: 334-342.
\end{abstract}

Pulmonary, Allergy, and Critical Care Division, University of Pennsylvania Medical Center, Philadelphia, PA, USA.

Correspondence: R.M. Kotloff, 838 West Gates, University of Pennsylvania Medical Center, 3400 Spruce Street, Philadelphia, PA 19104, USA.

Fax: 12156140869

E-mail: kotloff@mail.med.upenn.edu

Keywords: Immunosuppressive agents, lung transplantation, lymphoproliferative disorders, pneumonia

Received: April 162003

Accepted: April 232003

This work was supported by the Craig and Elaine Dobbin Pulmonary Research Fund of the University of Pennsylvania Medical Center, PA, USA.
Initial attempts to perform lung transplantation (LTx) were marred by technical limitations that often resulted in early postoperative death. Beginning in the 1980s, surgical advances, in conjunction with more effective immunosuppressive strategies, propelled the field forward and have made intermediateterm survival an achievable goal. While offering a vital therapeutic option for patients with advanced lung disease, LTx remains fraught with complications that threaten both the quality and duration of the recipient's life. In addition to allograft rejection (a topic covered elsewhere in this series), a multitude of medical complications mark the posttransplantation course. These complications, largely reflecting the consequences of global immunosuppression and drug toxicities, are the subject of this article.

\section{Infectious complications}

Infection is an ever-present threat to the well-being of the lung transplant recipient and is a leading cause of both early and late mortality. Infection rates among lung transplant recipients appear to be higher than those encountered in other solid organ transplant populations, likely related to the unique exposure of the lung allograft to the external environment and to the greater magnitude of immunosuppression employed $[1,2]$. In the following sections, the most common aetiological pathogens will be discussed; the reader seeking a more exhaustive discussion of the full spectrum of infections is referred to a recent review [3].

\section{Bacteria}

Bacterial infections of the lower respiratory tract comprise the majority of infectious complications and have a bimodal temporal distribution. Bacterial pneumonia is most frequently encountered in the first post-transplant month, with an incidence of $16 \%$ reported in a recent series [4]. In addition to the immunosuppressed status of the recipient, other factors that predispose to early bacterial pneumonias include the need for prolonged mechanical ventilatory support, blunted cough due to postoperative pain and weakness, disruption of lymphatics, and ischaemic injury to the bronchial mucosa with resultant impairment in mucociliary clearance. Although passive transfer of occult infection with the transplanted organ is an additional concern, the presence of organisms on Gram stain of donor bronchial washings is not predictive of subsequent pneumonia in the recipient [4]. Bacterial infections, in the form of purulent bronchitis, bronchiectasis and pneumonia, re-emerge as a late complication among patients who develop bronchiolitis obliterans syndrome (BOS; chronic rejection). Gram-negative pathogens, in particular Pseudomonas

Previous articles in this series: No. 1: Glanville AR, Estenne M. Indications, patient selection and timing of referral for lung transplantation. Eur Respir J 2003; 22: 845-852. No. 2: Boehler A, Estenne M. Post-transplant bronchiolitis obliterans. Eur Respir J 2003; 22: 1007-1018. No. 3: Knoop C, Haverich A, Fischer S. Immunosuppressive therapy after human lung transplantation. Eur Respir J 2003; 23 : 159-171. 
aeruginosa, are most frequently isolated in association with both early and late infectious events $[1,2]$.

Transplantation of patients with underlying cystic fibrosis (CF), whose native airways and sinuses are chronically infected with virulent bacterial pathogens, initially raised unique concerns about the potential excessive risk of postoperative infections. An extensive experience accrued to date in transplanting $\mathrm{CF}$ patients has generally allayed these concerns, demonstrating a rate of bacterial pulmonary infections similar to that encountered in other patient populations [5]. This favourable experience extends to $\mathrm{CF}$ patients who harbour panresistant strains of $P$. aeruginosa in their native airways [6]. In contrast, the presence of Burkholderia cepacia has been associated with a high risk of severe and often lethal postoperative infections, and consequently, with inferior survival rates. Two recent series from North American centres with extensive experience in transplantation of $\mathrm{CF}$ patients have documented 1-yr survival rates of $50 \%$ versus $83 \%$ and $67 \%$ versus $96 \%$ among CF patients with and without $B$. cepacia, respectively [7, 8]. Strain-specific virulence factors may dictate the behaviour of this organism. In particular, genomovar III has been associated with an excessive risk of lethal post-transplant infections [7].

\section{Cytomegalovirus}

Cytomegalovirus (CMV) is the most common viral pathogen encountered in the post-transplant period. Although the availability of effective antiviral therapy has significantly reduced the risk of death as a direct consequence of CMV infection, this virus continues to cause frequent, troubling infections, is associated with an increased risk of bacterial and fungal superinfections, and has been implicated as a possible risk factor in the development of BOS $[9,10]$. Infection in the recipient can occur following passive transmission of latent virus from the lung allograft or transfused blood products, or from reactivation of endogenous virus remotely acquired by the recipient. Those with primary infection (i.e. seronegative recipients who acquired infection from seropositive donors) are at greatest risk for developing severe, organ-invasive disease, particularly pneumonia [11].

CMV infection typically emerges $1-3$ months following transplantation, though onset may be delayed in patients receiving antiviral prophylaxis. Infection is often subclinical, evidenced only by silent viraemia or shedding of virus in the respiratory tract or urine. Clinical disease may present as a mononucleosis-like syndrome of fever, malaise and leukopaenia, or as organspecific invasion of the lung, gastrointestinal tract, central nervous system or retina. Detection of virus in peripheral blood by either the pp65 antigenaemia assay or by polymerase chain reaction (PCR) techniques establishes a diagnosis of CMV infection but does not necessarily reflect events at the tissue level. A diagnosis of CMV pneumonia, the most common manifestation of invasive disease in the lung transplant recipient, is unequivocally established only by demonstration of characteristic viral cytopathic changes on lung biopsy or on cytological specimens obtained by bronchoalveolar lavage (BAL) but, unfortunately, the sensitivity of these findings is relatively low. Caution must be exercised in interpretation of a positive rapid shell vial culture of BAL fluid, as shedding of virus into the respiratory tract can occur in the absence of tissue invasion. At best, a positive culture in the appropriate clinical setting provides only circumstantial support for the diagnosis of CMV pneumonia.

Standard treatment of CMV disease consists of a 2-3-week course of intravenous ganciclovir at a dose of $5 \mathrm{mg} \cdot \mathrm{kg}^{-1}$ twice daily, adjusted for renal insufficiency. Oral ganciclovir should not be used for definitive treatment due to its poor bioavailability and resultant low blood levels. Administration of oral valganciclovir appears to achieve blood levels comparable with that of intravenous ganciclovir, but its use cannot yet be endorsed in the absence of data confirming its efficacy in this setting [12]. Some experts advocate the addition of CMV hyperimmune globulin, particularly in cases of severe disease, but evidence supporting this practice is scant. Although treatment is generally effective, relapse rates of up to $60 \%$ in primary infection and $20 \%$ in previously exposed recipients have been reported in solid organ transplant recipients [13]. An additional concern is the emergence of ganciclovir-resistant strains of CMV. Recent reports document an incidence of $\sim 5 \%$ and identify an increased number of CMV episodes, increased cumulative exposure to ganciclovir, and use of antilymphocyte antibodies and daclizumab as possible risk factors [14, 15]. Foscarnet is the agent of choice for treatment of ganciclovir-resistant disease but its use is often limited by associated nephrotoxicity. The presence of ganciclovir-resistant disease is associated with decreased survival $[14,15]$.

In an attempt to minimise the adverse impact of CMV infection on the post-transplant course, emphasis has shifted to preventive strategies. The most effective strategy for seronegative recipients is the use of seronegative donors and screened blood products. While reducing the risk of CMV infection to a negligible level, this strategy results in increased waiting times for transplantation, as the majority of the donor pool is seropositive. A more practical strategy, now widely utilised when either donor or recipient is seropositive, is the initiation of a 3-month course of ganciclovir prophylaxis at the time of transplantation (universal prophylaxis) or with demonstration of asymptomatic viraemia by serial CMV pp65 antigen or PCR assays (pre-emptive prophylaxis). Interpretation of available studies is confounded by variation in duration of prophylaxis, route and frequency of administration, percentage of high-risk patients (donor-positive/recipientnegative) enrolled and duration of follow-up [16]. Taken in sum, these studies suggest that prophylaxis delays the onset of CMV infection and may decrease its incidence and severity [16-18]. Some studies have also suggested that prophylaxis delays the onset of BOS, but does not influence the frequency of this complication in the longer term [18, 19].

\section{Aspergillus}

Although a number of opportunistic and endemic fungi have been reported to cause pulmonary infections in lung transplant recipients, Aspergillus sp. are by far the most frequently encountered. As a ubiquitous organism acquired by inhalation, Aspergillus frequently colonises the airways of lung transplant recipients, with reported colonisation rates ranging 29-46\% [20-22]. Pretransplant colonisation with Aspergillus, a common phenomenon among patients with $\mathrm{CF}$, does not predispose to post-transplant colonisation [21]. In turn, post-transplantation airway colonisation does not appear to pose a major risk of subsequent progression to invasive disease. In this regard, data pooled from published studies suggest that only $3 \%$ of lung transplant recipients with airway colonisation progressed to invasive disease, although it is unclear how many of these patients received fungal prophylaxis in response to detection of the organism [20].

Aspergillus infections of the airway are encountered in $\sim 5 \%$ of lung transplant recipients $[2,20]$. The devitalised cartilage and foreign suture material of the fresh bronchial anastomosis create a nurturing environment for localised infection at the anastomotic site. A more diffuse ulcerative 
tracheobronchitis, with formation of pseudomembranes, is occasionally seen and likely follows severe ischaemic injury to the bronchial mucosa. Clustered within the first 6 months post-transplantation, these bronchial infections are usually asymptomatic and detected on surveillance bronchoscopy. Although usually responsive to oral itraconazole or to inhaled or intravenous amphotericin, airway infections have rarely progressed to invasive pneumonia or have resulted in fatal erosion into the adjacent pulmonary artery [20, 23, 24]. An increased risk of subsequent bronchial stenosis or bronchomalacia has also been reported [25], but it is unclear whether this is a consequence of the infection or of an underlying ischaemic injury to the bronchus that predisposed to infection.

Invasive aspergillosis is the most serious and life-threatening form of Aspergillus infection to plague the lung transplant recipient. An overall incidence of $5 \%$ has been calculated from pooled studies $[20,26]$. The majority of cases occur within the first post-transplant year. Most patients present with pneumonia, occasionally associated with disseminated infection. Symptoms of invasive aspergillosis are nonspecific and include fever, cough, pleuritic chest pain and haemoptysis. Radiographically, pulmonary aspergillosis may appear as single or multiple nodular or cavitary opacities or as alveolar consolidation. The "halo sign", a rim of ground-glass attenuation surrounding a central nodular opacity, is a highly suggestive but uncommon finding on chest computed tomography (CT).

Diagnosis of invasive pulmonary aspergillosis can be problematic. As discussed above, many lung transplant recipients are colonised with Aspergillus, making it difficult to interpret the significance of positive fungal stains and cultures derived from BAL specimens. Conversely, the sensitivity of bronchoscopic studies has been reported in the range of only $45-62 \%$ in solid organ transplant recipients with invasive disease [27]. Transthoracic needle aspiration can provide a diagnosis in some cases while surgical biopsy is reserved for cases that elude diagnosis with less invasive techniques.

Amphotericin B is considered the treatment of choice for invasive aspergillosis. Liposomal amphotericin preparations offer the advantage of decreased nephrotoxicity, an important feature for a drug used concurrently with nephrotoxic calcineurin inhibitors (cyclosporin A (CsA), tacrolimus (Tac)). Itraconazole has also been employed in the treatment of invasive aspergillosis. Advantages of this agent include the availability of an oral preparation for less ill, nonhospitalised patients, and the absence of nephrotoxicity. However, absorption of the oral formulations is suboptimal, and treatment failures can result from inadequate blood levels. As a potent inhibitor of the P450 enzyme system, the use of itraconazole can lead to dangerously high blood levels of CsA or Tac if appropriate adjustments in the dosing of these agents are not made. A new triazole, voriconazole, has recently been shown to have superior efficacy and less toxicity than amphotericin $\mathrm{B}$ in a large randomised trial of invasive aspergillosis in immunocompromised patients, and this agent may ultimately become the drug of choice [28]. Surgical resection of localised infection should be considered in cases refractory to antifungal therapy. Despite a multitude of treatment options, the composite mortality rate associated with invasive disease in published series involving lung transplant recipients approaches $60 \%[20]$.

The considerable morbidity and mortality associated with aspergillus infections have spurred interest in prophylactic measures. Some centres employ universal prophylaxis in the early post-transplant period with either aerosolised amphotericin B or oral itraconazole, while others selectively administer these agents only to those patients whose airways become colonised with Aspergillus. Although several observational studies employing historical controls suggest a possible reduction in infection rates in association with prophylactic measures, the absence of randomised, controlled trials precludes firm endorsement of these strategies [29].

\section{Neoplastic complications}

\section{Post-transplant lymphoproliferative disease}

Post-transplant lymphoproliferative disease (PTLD) is a term applied to a spectrum of abnormal B-cell proliferative responses ranging from benign polyclonal hyperplasia to more commonly encountered malignant lymphomas. The incidence of PTLD in the lung transplant population ranges $1.8-7.9 \%$ in single-centre case series, with an overall pooled incidence of $4.9 \%$ [30-32]. Among the myriad neoplasms that arise following transplantation, PTLD is second in frequency only to nonmelanoma skin cancers.

Epstein-Barr virus (EBV) has been identified as the stimulus for B-cell proliferation, which proceeds in an unchecked fashion due to the muted cytotoxic T-cell response in the immunosuppressed host. EBV-naive recipients who acquire primary infection at the time of organ transplantation are at greatest risk of developing PTLD. In one series, the incidence of PTLD was 33\% among recipients who were seronegative prior to transplant, compared with only $1.7 \%$ for those who were previously exposed [30]. The use of antilymphocyte antibodies has also been associated with an increased risk of PTLD, likely reflecting the profound impact of these agents on intrinsic T-cell activity [33].

The incidence of PTLD is greatest within the first posttransplantation year and the mode of presentation of these early onset cases is distinct from that of late-onset cases [32, 34]. In this regard, the majority of early onset cases involve the allograft, typically presenting as one or more pulmonary nodules that may be accompanied by mediastinal adenopathy. In contrast, intra-abdominal and disseminated forms of disease predominate in cases presenting beyond the first year. The diagnosis of PTLD is most firmly established by tissue biopsy, although fine needle aspiration may occasionally yield sufficient material to make a cytological diagnosis. Care must be exercised in interpreting transbronchial lung biopsies, as the aggregates of lymphocytes associated with acute cellular rejection can appear similar to foci of PTLD on these small tissue specimens. Demonstration of the presence of EBV-infected cells by in situ hybridisation or immunohistochemical staining can help to confirm a diagnosis in difficult cases. Determination of EBV viral load in the peripheral blood using deoxyribonucleic acid amplification techniques has been touted as an ancillary diagnostic tool. Two preliminary studies involving lung transplant recipients suggest that an elevated viral load correlates with the presence of PTLD [35, 36], although the specificity of this finding has been questioned [37]. Additional studies employing uniform assay techniques and threshold values for positive results are required before this test can be advocated.

Initial treatment focuses on reduction in the magnitude of immunosuppression to permit partial restoration of host cellular immunity directed toward EBV. Patients whose disease is confined to the allograft are most likely to respond to this measure, but there is an attendant risk of precipitating acute or chronic rejection with this approach. Use of rituximab, a chimeric human-mouse monoclonal antibody directed against the B-cell marker CD20, has been associated with complete tumour regression and minimal side-effects [38-40]. It offers an attractive option for patients with more aggressive disease and those who fail or cannot tolerate a reduction in immunosuppression. Standard chemotherapy 
has been employed for refractory cases but the associated neutropaenia is poorly tolerated by a patient population already profoundly immunosuppressed. There is no proven role for antiviral therapy in the setting of established PTLD, although there is preliminary evidence that the prophylactic use of antiviral agents initiated prior to the rapid replication phase of infection may reduce the subsequent risk of developing PTLD [31, 41]. Finally, the technique of adoptive immunotherapy, involving in vitro expansion and subsequent reinfusion of recipient EBV-specific cytotoxic T-cells, holds promise as a novel means of treating PTLD [42].

Mortality related to PTLD has been reported in the range of $37-50 \%[32,34]$. Early onset disease and disease restricted to the allograft are associated with a more benign course. In contrast, disseminated disease carries a much graver prognosis $[32,43]$.

\section{Lung cancer}

The development of lung cancer following LTx has been reported exclusively in patients with underlying chronic obstructive pulmonary disease (COPD) or pulmonary fibrosis, both of which predispose to lung cancer. Additionally, the majority of these patients have significant smoking histories. The reported incidence of lung cancer following transplantation is $2.0-3.7 \%$ in patients with chronic obstructive lung disease and $3.4-4.0 \%$ for the pulmonary fibrosis population $[44,45]$. Data are conflicting on whether transplantation confers an increased likelihood of developing this form of cancer or whether the incidence is comparable with that of the general population with similar risk factors [46]. Lung cancer may complicate the transplant course in several ways. Previously unsuspected cancer may be incidentally detected in the explanted lung at the time of transplantation, placing the recipient at risk for subsequent recurrence [44]. On occasion, LTx has been performed as definitive treatment for underlying bronchoalveolar carcinoma; a high rate of recurrence in the allografts has been documented in these circumstances [47]. Cancer may also arise de novo in the remaining native lung following single LTx. Lung cancer in the transplant recipient can progress in a rapid fashion over a short period of time, mimicking an infectious process [44]. This aggressive behaviour may reflect loss of antitumour immune surveillance in the immunosuppressed host or may be due to a more specific effect of CsA in promoting tumour growth [48].

\section{Osteoporosis}

Osteoporosis is characterised by low bone mass accompanied by microarchitectural changes that increase the susceptibility to fracture. It is operationally defined as a bone mineral density (BMD) exceeding 2.5 sDs below the mean for young adults; a BMD between 1.0-2.5 SDs below the mean defines osteopaenia. A number of studies have now documented a high risk of progressive bone demineralisation and an accompanying risk of fractures among lung transplant recipients. In a prospective study of 28 patients treated only with calcium and vitamin D supplementation, SPIRA et al. [49] demonstrated a loss of BMD of $\sim 5 \%$ within the first posttransplant year, with an overall increase in the prevalence of osteoporosis from $54 \%$ pretransplantation to $78 \%$ and a posttransplantation fracture rate of $18 \%$. In another study, $73 \%$ of lung transplant recipients had BMD that was at or beyond the fracture threshold and $28 \%$ of patients sustained fractures [50]. Osteoporosis-induced fractures can be painful and debilitating, with the potential to compromise the outcome of transplantation by rendering a patient nonambulatory or narcotic-dependent despite excellent graft function.

Pre-existing bone disease contributes significantly to posttransplantation osteoporosis and fracture risk. The prevalence of bone demineralisation is remarkably high among patients with advanced lung disease of diverse aetiologies who are listed for LTx $[49,51]$. For example, osteoporosis rates of $41-67 \%$ in the CF population and $36-69 \%$ among patients with advanced COPD have been reported and most of those without frank osteoporosis are osteopaenic [51-53]. Risk factors for bone demineralisation among patients with advanced lung disease include low body mass index, corticosteroid use, smoking and diminished weight-bearing activity. Hypogonadism, resulting from chronic illness and from glucocorticoidinduced suppression of sex hormone production, is an underappreciated factor contributing to accelerated bone loss. Finally, vitamin D deficiency has been implicated in both the $\mathrm{CF}$ and $\mathrm{COPD}$ populations $[52,53]$.

Immunosuppressive drugs are cited as a second major cause of progressive bone demineralisation following transplantation. Pre-eminent among these are corticosteroids, which are employed in high doses for the first several post-transplant months and then tapered to a lower maintenance dose that is typically maintained indefinitely. In one prospective study of lung transplant recipients, a significant correlation was identified between cumulative steroid dose post-transplant and net decline in BMD [49]. The most rapid period of bone demineralisation occurs within the first 6 months of initiation of these agents. Corticosteroids exert their deleterious effects through complex mechanisms that result in both inhibition of osteoblast activity and activation of osteoclasts, resulting in net bone resorption [49]. Additionally, these drugs reduce intestinal absorption of calcium, enhance urinary calcium excretion, and impair production of oestrogen and testosterone. The link between other immunosuppressive agents and bone loss is less well defined. In an animal model, administration of CsA and Tac resulted in the rapid development of severe bone loss [54, 55], but studies in humans have thus far failed to establish an association [49]. Data on the effects of rapamycin (sirolimus) on bone metabolism are conflicting. Rapamycin enhances osteoclastogenesis and osteoclastic resorption activity in vitro but does not result in bone loss in an animal model $[54,56]$. Its effect in humans is uncertain.

All patients with advanced lung disease presenting for transplant evaluation should be screened for the presence of bone loss by dual x-ray absorpiometry (DEXA). The protracted waiting period to transplantation provides an opportunity to intervene and perhaps reduce the risk of posttransplantation complications. Recommendations for prevention and treatment of osteoporosis in lung transplant candidates and recipients are based on guidelines established by the American College of Rheumatology for the more generic problem of glucocorticoid-induced bone loss [57]. All patients should receive calcium $\left(1,000-1,500 \mathrm{mg} \cdot \mathrm{day}^{-1}\right)$ and vitamin D (400-800 IU $\cdot$ day $\left.^{-1}\right)$ supplementation, a regimen that has been shown to preserve BMD in patients with normal bone mass receiving chronic low-dose corticosteroid therapy [53]. Owing to malabsorption problems, measurement of serum 25-hydroxyvitamin D levels is necessary in CF patients to ensure that doses sufficient to achieve normal levels are administered [52]. Hormone replacement therapy is recommended for postmenopausal females without contraindications. Its use must be balanced against the risks associated with this treatment and the availability of alternative agents such as raloxifene, a selective oestrogen-receptor modulator that has oestrogen-like effects on bone but does not appear to increase the risk of breast or endometrial cancer. Males with low testosterone levels should receive replacement therapy, an 
intervention shown to modestly improve BMD in one small study of glucocorticoid-treated asthmatic males [58].

Bisphosphonates form the cornerstone of antiresorptive therapy for prophylaxis and treatment of osteoporosis. The ability of these agents to improve BMD and decrease fracture rates in patients receiving corticosteroids has been demonstrated in several randomised, controlled trials [57, 59, 60]. More limited data exist supporting the benefits of bisphosphonates in the lung transplant population. A prospective, randomised, controlled trial comparing the efficacy of pamidronate, calcium and vitamin $\mathrm{D}$ with calcium and vitamin $\mathrm{D}$ alone, in CF patients with osteoporosis who had undergone LTx, demonstrated greater BMD at the end of $2 \mathrm{yrs}$ in the pamidronate-treated group but no difference in fracture rates [61]. A second prospective, uncontrolled trial involving 45 lung transplant recipients showed that the addition of pamidronate to calcium, vitamin $\mathrm{D}$ and hormonal replacement therapy (as indicated), stabilised BMD more effectively and reduced the rate of fractures compared with historical controls [62]. Based on this data and extrapolating from the more extensive data derived from the nontransplant population, it appears reasonable to administer bisphosphonate therapy to all transplant recipients with osteopaenia or osteoporosis, and to patients with normal BMD in whom corticosteroids are being newly initiated [57]. Those patients who have maintained normal BMD despite chronic steroid administration can be followed with serial DEXA scans, with treatment reserved for documented decline in BMD. Since bisphosphonates are renally excreted, they should be used with caution in patients with significant renal insufficiency. Patients unable to take the oral preparations due to significant gastro-oesophageal reflux disease can receive intermittent intravenous pamidronate.

Calcitonin is an alternative antiresorptive agent. Studies in glucocorticoid-treated patients have not consistently demonstrated benefit [53] and there is a notable paucity of data in the transplant population. Thus, its use should be restricted to patients unable to tolerate or unwilling to take bisphosphonate therapy. A newly available treatment of osteoporosis is parathyroid hormone, which works by stimulating bone formation rather than by inhibiting resorption. In postmenopausal females, this agent has been shown to significantly increase BMD and reduce fracture rates [63]. Additional studies are required to determine if this approach will benefit patients with advanced lung disease and transplant recipients. Finally, for patients with painful vertebral compression fractures, vertebroplasty has been associated with rapid relief of pain in $67-100 \%$ of cases [64].

\section{Gastrointestinal complications}

A broad spectrum of gastrointestinal complications has been reported following LTx. Many of the reported complications, such as peptic ulcer disease, gastritis and pseudomembranous colitis, are generic to all surgical populations. In contrast, CsA-induced gastroparesis and CMV infection of the gastrointestinal tract are examples of complications unique to the transplant population. In one clinical series of lung transplant recipients, a 50\% incidence of gastrointestinal complications was documented, although the majority of reported complications were relatively minor and resolved with conservative management. The one notable exception was bowel perforation, documented in $10 \%$ of patients and associated with a $75 \%$ mortality rate [65].

Recent observations suggesting a link between gastrooesophageal reflux disease and chronic allograft rejection have generated both interest and debate. BERKOWITZ et al.
[66] found that four of nine (44\%) lung transplant recipients with documented gastroparesis developed bronchiolitis obliterans compared with only six of $29(21 \%)$ without this disorder. Furthermore, food particles were detected in BAL fluid from two of the four patients with gastroparesis and graft dysfunction, implicating microaspiration as the mechanism. PALMER et al. [67] described a transplant recipient with severe gastro-oesophageal reflux disease who developed a rapid and sustained decline in lung function that was attributed to BOS. Transbronchial biopsies revealed bronchiolar inflammation. Owing to concerns that reflux could be a contributing factor, the patient underwent Nissen fundoplication, following which there was a dramatic improvement in lung function and resolution of histological evidence of airway inflammation. In a follow-up study from the same transplant centre, 18 lung transplant recipients with severe reflux disease, 11 of whom had BOS, underwent fundoplication surgery [68]. Twelve of the 18 patients demonstrated improvement in forced expiratory volume in one second, although the magnitude of individual improvements was not reported. In light of the anecdotal and circumstantial nature of the evidence, firm conclusions about the relationship between reflux disease and allograft dysfunction cannot be made. Further studies are required before early surgical intervention to correct reflux can be endorsed.

\section{Neurological complications}

\section{Leukoencephalopathy}

Neurological complications occur in up to one-quarter of lung transplant recipients [69]. Excluding central nervous system infections, the majority of complications represent adverse effects of the calcineurin inhibitors. Most common among these are tremor, headache and parasthesias. Of more concern is drug-induced leukoencephalopathy. The manifestations of this disorder are protean and include confusion, coma, aphasia, seizures, quadraplegia and cortical blindness [70-74]. CT imaging of the brain demonstrates nonenhancing areas of low attenuation in the cerebral white matter, while on magnetic resonance imaging, the more sensitive of the two techniques, these abnormalities appear as areas of increased signal on T2-weighted images. There is a predilection for involvement of the occipital lobes. The mechanism of injury remains speculative; both a direct toxic injury and druginduced vasospasm with secondary ischaemic injury have been suggested. Factors associated with the occurrence of leukoencephalopathy include hypomagnesaemia, low serum cholesterol levels, administration of high doses of corticosteroids and hypertension [73, 74], but these observed associations have failed to shed light on the underlying pathogenesis. Patients may develop this complication despite therapeutic drug levels. Treatment consists of dose reduction or discontinuation of the responsible agent, following which neurological and radiographical abnormalities promptly improve. Curiously, case reports document successful substitution of the alternative calcineurin inhibitor for the one that presumably caused leukoencephalopathy, without relapse of the neurological syndrome [71, 75]. Brain biopsy to exclude disorders such as lymphoma or progressive multifocal leukoencephalopathy, should be considered in patients who fail to improve with manipulation of immunosuppressive medications.

\section{Stroke}

Stroke has been reported to occur with an incidence of 5\% in the lung transplant population [69]. In addition to the more 
common atherothrombotic mechanism of stroke, two embolic mechanisms unique to this patient population must be considered. Failure to fully de-gas the pulmonary vascular tree and cardiac chambers after completion of the vascular anastomoses can lead to air embolism in the immediate postoperative period. Alternatively, formation of thrombus in the region of the left atrial anastomosis or pulmonary veins can result in embolic stroke days to weeks after transplantation [76]. Demonstration of multiple areas of brain infarction, in a pattern consistent with embolic stroke, should prompt performance of transoesophageal echocardiography and, if thrombus is documented, systemic anticoagulation should be instituted in the absence of contraindications.

\section{Hyperammonaemia}

Severe idiopathic hyperammonaemia has been reported as a rare cause of coma in the early postoperative period following LTx [77]. Antecedent major abdominal complications (e.g. bowel perforation), use of total parenteral nutrition, and underlying primary pulmonary hypertension have been associated with an increased risk of developing this complication [77]. Hyperammonaemia in this setting is not a consequence of fulminant hepatic failure. Rather, detailed metabolic analysis of the livers of two patients who died from this complication suggests that a deficiency in hepatic glutamine synthetase, a urea cycle enzyme crucial to the handling of nitrogenous wastes, underlies the pathogenesis of this disorder [78]. Lethargy, agitation and seizures herald the onset of hyperammonaemia. Coma due to cerebral oedema and increased intracranial pressure rapidly ensues. A multipronged approach to treatment has been advocated, with discontinuation of exogenous nitrogen sources, initiation of haemodialysis to clear ammonia from the bloodstream, and administration of the ammonia-trapping agents sodium phenylacetate and sodium benzoate [79]. Successful recovery with early institution of these measures has been reported but the majority of reported cases have ended in death.

\section{Renal complications}

\section{Calcineurin-inhibitor nephrotoxicity}

In addition to its intended immunosuppressive effect, administration of the calcineurin inhibitors is associated with the nearly universal untoward consequence of nephrotoxicity. In a study of 219 lung and heart-lung transplant recipients receiving a calcineurin inhibitor-based regimen, $91 \%$ of patients experienced a decrease in renal function by 6 months. A doubling of serum creatinine was noted in $34 \%$ of patients at $1 \mathrm{yr}, 43 \%$ at $2 \mathrm{yrs}$ and $53 \%$ at $5 \mathrm{yrs} ; 4.6 \%$ of patients ultimately developed renal failure requiring haemodialysis [80]. Factors predictive of nephrotoxicity include the serum creatinine at 1 month post-transplant and the cumulative duration of diastolic blood pressure exceeding $90 \mathrm{mmHg}[80,81]$.

Nephrotoxicity of the calcineurin inhibitors has been most extensively studied in association with CsA. Two forms of injury have been described, acute and chronic [82]. Acute nephrotoxicity is a result of intense vasoconstriction of preglomerular (afferent) arterioles, leading to a decrease in renal blood flow and glomerular filtration rate. These effects are dose-related and reversible upon withdrawal of CsA. Putative mechanisms of CsA-induced vasoconstriction include enhanced production of endothelin-1, thromboxane and renin-angiotensin, activation of the sympathetic nervous system and inhibition of nitric oxide production. Chronic nephrotoxicity is marked by structural changes in renal architecture, including tubular atrophy, tubulointerstitial fibrosis and glomerulosclerosis [82]. This form of injury is typically progressive over time, leading to permanent impairment in renal function. Whether there is a partially reversible component with cessation of the drug is unclear, since in the absence of serial biopsies it is difficult to discern the degree to which any observed improvement in renal function is due to attenuation of structural derangements versus resolution of CsA-induced vasoconstriction. Several CsA-mediated effects may be operative in causing the observed structural injury: 1) renal ischaemia due to sustained vasoconstriction; 2) upregulation of the profibrotic cytokine, transforming growth factor- $\beta$; and 3 ) increased apoptosis of tubular and interstitial cells [82].

Strategies to minimise the risk of severe nephrotoxicity and renal failure are evolving. Patients who experience a significant rise in creatinine within the first 6 months posttransplantation are at particular risk and should be targeted for early intervention. Preliminary data also suggest that elevated excretion of retinal-binding protein in the urine, a marker of subclinical proximal tubular dysfunction, may identify patients at high risk for severe nephrotoxicity and may allow for intervention even before a significant loss of renal function has occurred [83]. Aggressive blood pressure control is warranted for all transplant recipients, in light of the epidemiological evidence linking diastolic hypertension with more severe renal insufficiency [80]. Some have advocated the preferential use of calcium-channel blockers to treat hypertension, based on the ability of these agents to counteract the acute vasoconstrictive effects of calcineurin inhibitors. However, a long-term protective effect of these agents has not been demonstrated [80, 84]. For patients who develop significant $\mathrm{CsA}$-induced nephrotoxicity, conflicting data exist on the merits of conversion to Tac. Numerous large randomised trials comparing $\mathrm{CsA}$ and Tac as primary immunosuppression in solid organ transplant populations detected equivalent nephrotoxic effects [80]. Conversely, administration of Tac to normal volunteers was not associated with the acute renal vasoconstrictive effects seen with CsA [85]. Moreover, several small case series involving organ transplant recipients documented improvement in renal function following elective conversion from CsA to Tac in the setting of nephrotoxicity [86-88]. Finally, use of Tac in the first 6 months after LTx was associated with a reduced risk of nephrotoxicity by multivariate analysis [80]. Pending further clarification of this issue, conversion to Tac appears reasonable as a first step in addressing CsA nephrotoxicity. An alternative approach in patients with either CsA- or Tac-associated nephrotoxicity is the administration of rapamycin (sirolimus), a novel immunosuppressive agent whose mechanism of action is independent of calcineurin blockade and whose use is not associated with significant nephrotoxicity. Employed either as a substitute for calcineurin inhibitors, or in conjunction with reduced doses of these agents, rapamycin has been reported to facilitate recovery of renal function in patients with nephrotoxicity [89].

\section{Haemolytic uraemic syndrome}

A rare but potentially devastating renal complication affecting transplant recipients is the haemolytic uraemic syndrome (HUS). Development of HUS is considered to be an adverse effect of calcineurin inhibitor administration and has been reported in association with both CsA and Tac [90-93]. Evidence supporting a causal role for these agents is 
scant, however, and includes the reappearance of HUS upon rechallenge with the drug in question [93], and the reproduction of this syndrome in CsA-treated animals, usually in association with a second insult [94]. Mechanisms by which these drugs could produce HUS include direct injury to the renal endothelium and a shift in the balance of thromboxane and prostaglandin $\mathrm{I}_{2}$ production favouring platelet aggregation.

The incidence of HUS in the lung transplant population is unknown; documentation is largely at a case report level [92, 95]. In a recent review of 91 solid organ recipients with HUS, only one case involved a lung transplant recipient, although this may simply reflect the relatively small size of the lung transplant population compared with other solid organ groups [96]. The diagnosis is established principally on the basis of laboratory data revealing worsening azotaemia, thrombocytopaenia, and microangiopathic haemolytic anaemia signified by an elevated lactate dehydrogenase level and the presence of schistocytes on peripheral smear. Treatment involves discontinuation of the calcineurin inhibitor and, in refractory or severe cases, initiation of plasmapheresis. Case reports suggest that the calcineurin inhibitors are not necessarily cross-reactive in producing this syndrome and substitition with the alternative agent is therefore an appropriate strategy [90, 92]. Rapamycin has not been implicated, to date, in the development of HUS and offers an additional immunosuppressive option in lieu of the calcineurin inhibitors.

\section{Future directions}

As detailed in this article, current immunosuppressive strategies subject the lung transplant recipient to a high risk of infection, neoplasm and adverse drug toxicities. New agents with a more favourable side-effect profile are being developed, but as long as global immunosuppression is the intended consequence, the infectious and neoplastic risks will remain. A more desirable goal under active investigation is the induction of immune tolerance, whereby the immune system is manipulated to selectively and permanently accept the allograft without the need for lifelong administration of immunosuppressive agents. Tolerance has been achieved in animal models but remains an elusive goal in human transplantation. When ultimately realised, the strategy of immune tolerance will hopefully provide lung transplant recipients with the promise of prolonged, complication-free survival.

\section{References}

1. Maurer JR, Tullis E, Grossman RF, Vellend H, Winton TL, Patterson GA. Infectious complications following isolated lung transplantation. Chest 1992; 101: 1056-1059.

2. Kramer MR, Marshall SE, Starnes VA, Gamberg P, Amitai Z, Theodore J. Infectious complications in heart-lung transplantation. Analysis of 200 episodes. Arch Intern Med 1993; 153: 2010-2016.

3. Alexander BD, Tapson VF. Infectious complications of lung transplantation. Transplant Infect Dis 2001; 3: 128-137.

4. Weill D, Dey GC, Hicks RA, et al. A positive donor gram stain does not predict outcome following lung transplantation. J Heart Lung Transplant 2002; 21: 555-558.

5. Flume PA, Egan TM, Paradowski LJ, Detterbeck FC, Thompson JT, Yankaskas JR. Infectious complications of lung transplantation. Impact of cystic fibrosis. Am J Respir Crit Care Med 1994; 149: 1601-1607.

6. Aris RM, Gilligan PH, Neuringer IP, Gott KK, Rea J, Yankaskas JR. The effects of panresistant bacteria in cystic fibrosis patients on lung transplant outcome. Am J Respir Crit Care Med 1997; 155: 1699-1704.

7. Aris RM, Routh JC, LiPuma JJ, Heath DG, Gilligan PH. Lung transplantation for cystic fibrosis patients with Burkholderia cepacia complex. Survival linked to genomovar type. Am J Respir Crit Care Med 2001; 164: 2102-2106.

8. Chaparro C, Maurer J, Gutierrez C, et al. Infection with Burkholderia cepacia in cystic fibrosis: outcome following lung transplantation. Am J Respir Crit Care Med 2001; 163: 43-48.

9. Duncan SR, Paradis IL, Yousem SA, et al. Sequelae of cytomegalovirus pulmonary infections in lung allograft recipients. Am Rev Respir Dis 1992; 146: 1419-1425.

10. Estenne M, Maurer JR, Boehler A, et al. Bronchiolitis obliterans syndrome 2001: an update of the diagnostic criteria. J Heart Lung Transplant 2002; 21: 297-310.

11. Ettinger NA, Bailey TC, Trulock EP, et al. Cytomegalovirus infection and pneumonitis: impact after isolated lung transplantation. Am Rev Respir Dis 1993; 147: 1017-1023.

12. Martin DF, Sierra-Madero J, Walmsley S, et al. A controlled trial of valganciclovir as induction therapy for cytomegalovirus retinitis. $N$ Engl J Med 2002; 346: 1119-1126.

13. Rubin RH. Prevention and treatment of cytomegalovirus disease in heart transplant patients. $J$ Heart Lung Transplant 2000; 19: 731-735.

14. Bhorade SM, Lurain NS, Jordan A, et al. Emergence of ganciclovir-resistant cytomegalovirus in lung transplant recipients. J Heart Lung Transplant 2002; 21: 1274 1282.

15. Kruger RM, Shannon WD, Arens MQ, Lynch JP, Storch GA, Trulock EP. The impact of ganciclovir-resistant cytomegalovirus infection after lung transplantation. Transplantation 1999; 68: 1272-1279.

16. Zamora MR. Controversies in lung transplantation: management of cytomegalovirus infections. J Heart Lung Transplant 2002; 21: 841-849.

17. Hertz MI, Jordan C, Savik SK, et al. Randomized trial of daily versus three-times-weekly prophylactic ganciclovir after lung and heart-lung transplantation. J Heart Lung Transplant 1998; 17: 913-920.

18. Soghikian MV, Valentine VG, Berry GJ, Patel HR, Robbins RC, Theodore J. Impact of ganciclovir prophylaxis on heart-lung and lung transplant recipients. J Heart Lung Transplant 1996; 15: 881-887.

19. Duncan SR, Grgurich WF, Iacono AT, et al. A comparison of ganciclovir and acyclovir to prevent cytomegalovirus after lung transplantation. Am J Respir Crit Care Med 1994; 150: $146-152$.

20. Mehrad B, Paciocco G, Martinez FJ, Ojo TC, Iannettoni MD, Lynch JP 3rd. Spectrum of Aspergillus infection in lung transplant recipients: case series and review of the literature. Chest 2001; 119: 169-175.

21. Paradowski LJ. Saprophytic fungal infections and lung transplantation-Revisited. J Heart Lung Transplant 1997; 16: 524-531.

22. Cahill B, Hibbs JR, Savik K, et al. Aspergillus airway colonization and invasive disease after lung transplantation. Chest 1997; 112: 1160-1164.

23. Kessler R, Massard G, Warter A, Wihlm JM, Weitzenblum E. Bronchial-pulmonary artery fistula after unilateral lung transplantation: a case report. J Heart Lung Transplant 1997; 16: 674-677.

24. Birsan T, Taghavi S, Klepetko W. Treatment of aspergillusrelated ulcerative tracheobronchitis in lung transplant recipients. J Heart Lung Transplant 1998; 17: 437-438.

25. Nunley DR, Gal AA, Vega JD, Perlino C, Smith P, Lawrence EC. Saprophytic fungal infections and complications involving the bronchial anastomosis following human lung transplantation. Chest 2002; 122: 1185-1191.

26. Gordon SM, Avery RK. Aspergillosis in lung transplantation: incidence, risk factors, and prophylactic strategies. Transpl Infect Dis 2001; 3: 161-167. 
27. Paterson DL, Singh N. Invasive aspergillosis in transplant recipients. Medicine (Baltimore) 1999; 78: 123-138.

28. Herbrecht R, Denning DW, Patterson TF, et al. Voriconazole versus amphotericin B for primary therapy of invasive aspergillosis. $N$ Engl J Med 2002; 347: 408-415.

29. Reichenspurner $\mathrm{H}$, Gamberg $\mathrm{P}$, Nitschke $\mathrm{M}$, et al. Significant reduction in the number of fungal infections after lung-, heart-lung, and heart transplantation using aerosolized amphotericin B prophylaxis. Transplant Proc 1997; 29: 627-628.

30. Aris RM, Maia DM, Neuringer IP, et al. Post-transplantation lymphoproliferative disorder in the Epstein-Barr virus-naive lung transplant recipient. Am J Respir Crit Care Med 1996; 154: 1712-1717.

31. Levine SM, Angel L, Anzueto A, et al. A low incidence of posttransplant lymphoproliferative disorder in 109 lung transplant recipients. Chest 1999; 116: 1273-1277.

32. Paranjothi S, Yusen RD, Kraus MD, Lynch JP, Patterson GA, Trulock EP. Lymphoproliferative disease after lung transplantation: comparison of presentation and outcome of early and late cases. J Heart Lung Transplant 2001; 20: 1054-1063.

33. Swinnen LJ, Costanzo-Nordin MR, Fisher SG, et al. Increased incidence of lymphoproliferative disorder after immunosuppression with the monoclonal antibody OKT3 in cardiac-transplant recipients. N Engl J Med 1990; 323: 17231728 .

34. Armitage JM, Kormos RL, Stuart RS, et al. Posttransplant lymphoproliferative disease in thoracic organ transplant patients: ten years of cyclosporine-based immunosuppression. J Heart Lung Transplant 1991; 10: 877-887.

35. Stevens SJ, Verschuuren EA, Pronk I, et al. Frequent monitoring of Epstein-Barr virus DNA load in unfractionated whole blood is essential for early detection of posttransplant lymphoproliferative disease in high-risk patients. Blood 2001; 97: 1165-1171.

36. Tsai DE, Nearey M, Hardy CL, et al. Use of EBV PCR for the diagnosis and monitoring of post-transplant lymphoproliferative disorder in adult solid organ transplant patients. Am J Transplant 2002; 2: 946-954.

37. Green M, Webber SA. EBV viral load monitoring: unanswered questions. Am J Transplant 2002; 2: 894-895.

38. Reynaud-Gaubert M, Stoppa AM, Gaubert J, Thomas P, Fuentes P. Anti-CD20 monoclonal antibody therapy in Epstein-Barr Virus-associated B cell lymphoma following lung transplantation. J Heart Lung Transplant 2000; 19: 492495.

39. Verschuuren EA, Stevens SJ, van Imhoff GW, et al. Treatment of posttransplant lymphoproliferative disease with rituximab: the remission, the relapse, and the complication. Transplantation 2002; 73: 100-104.

40. Niedermeyer J, Hoffmeyer F, Hertenstein B, Hoeper MM, Fabel H. Treatment of lymphoproliferative disease with rituximab. Lancet 2000; 355: 499

41. Malouf MA, Chhajed PN, Hopkins P, Plit M, Turner J, Glanville AR. Anti-viral prophylaxis reduces the incidence of lymphoproliferative disease in lung transplant recipients. J Heart Lung Transplant 2002; 21: 547-554.

42. Khanna R, Bell S, Sherritt M, et al. Activation and adoptive transfer of Epstein-Barr virus-specific cytotoxic $\mathrm{T}$ cells in solid organ transplant patients with posttransplant lymphoproliferative disease. Proc Natl Acad Sci USA 1999; 96: 10391-10396.

43. Tsai DE, Hardy CL, Tomaszewski JE, et al. Reduction in immunosuppression as initial therapy for posttransplant lymphoproliferative disorder: analysis of prognostic variables and long-term follow-up of 42 adult patients. Transplantation 2001; 71: 1076-1088.

44. Arcasoy SM, Hersh C, Christie JD, et al. Bronchogenic carcinoma complicating lung transplantation. J Heart Lung Transplant 2001; 20: 1044-1053.

45. Collins J, Kazerooni EA, Lacomis J, et al. Bronchogenic carcinoma after lung transplantation: frequency, clinical characteristics, and imaging findings. Radiology 2002; 224: 131-138.

46. Penn I. Posttransplant malignancies. Transplant Proc 1999; 31: $1260-1262$.

47. Garver RI Jr, Zorn GL, Wu X, McGiffin DC, Young KR Jr, Pinkard NB. Recurrence of bronchioloalveolar carcinoma in transplanted lungs. N Engl J Med 1999; 340: 1071-1074.

48. Hojo M, Morimoto $\mathrm{T}$, Maluccio $\mathrm{M}$, et al. Cyclosporine induces cancer progression by a cell-autonomous mechanism. Nature 1999; 397: 530-534.

49. Spira A, Gutierrez C, Chaparro C, Hutcheon MA, Chan CK. Osteoporosis and lung transplantation: a prospective study. Chest 2000; 117: 476-481.

50. Aris RM, Neuringer IP, Weiner MA, Egan TM, Ontjes D. Severe osteoporosis before and after lung transplantation. Chest 1996; 109: 1176-1183.

51. Tschopp O, Boehler A, Speich R, et al. Osteoporosis before lung transplantation: association with low body mass index, but not with underlying disease. Am J Transplant 2002; 2: $167-172$

52. Donovan DS Jr, Papadopoulos A, Staron RB, et al. Bone mass and vitamin D deficiency in adults with advanced cystic fibrosis lung disease. Am J Respir Crit Care Med 1998; 157: 1892-1899.

53. Biskobing DM. COPD and osteoporosis. Chest 2002; 121: 609-620.

54. Romero DF, Buchinsky FJ, Rucinski B, et al. Rapamycin: a bone sparing immunosuppressant? J Bone Miner Res 1995; 10: $760-768$

55. Cvetkovic M, Mann GN, Romero DF, et al. The deleterious effects of long-term cyclosporine A, cyclosporine $\mathrm{G}$, and FK506 on bone mineral metabolism in vivo. Transplantation 1994; 57: 1231-1237.

56. Shui C, Riggs BL, Khosla S. The immunosuppressant rapamycin, alone or with transforming growth factor-b, enhances osteoclast differentiation of RAW264.7 monocytemacrophage cells in the presence of RANK-ligand. Calcif Tissue Int 2002; 71: 437-446.

57. American College of Rheumatology Ad Hoc Committee on Glucocorticoid-Induced Osteoporosis. Recommendations for the prevention and treatment of glucocorticoid-induced osteoporosis: 2001 update. Arthritis Rheum 2001; 44: 14961503.

58. Reid IR, Wattie DJ, Evans MC, Stapleton JP. Testosterone therapy in glucocorticoid-treated men. Arch Intern Med 1996; 156: 1173-1177.

59. Adachi JD, Bensen WG, Brown $\mathbf{J}$, et al. Intermittent etidronate therapy to prevent corticosteroid-induced osteoporosis. N Engl J Med 1997; 337: 382-387.

60. Saag KG, Emkey R, Schnitzer TJ, et al. Alendronate for the prevention and treatment of glucocorticoid-induced osteoporosis. Glucocorticoid-Induced Osteoporosis Intervention Study Group. N Engl J Med 1998; 339: 292-299.

61. Aris RM, Lester GE, Renner JB, et al. Efficacy of pamidronate for osteoporosis in patients with cystic fibrosis following lung transplantation. Am J Respir Crit Care Med 2000; 162: 941-946.

62. Cahill BC, O'Rourke MK, Parker S, Stringham JC, Karwande SV, Knecht TP. Prevention of bone loss and fracture after lung transplantation: a pilot study. Transplantation 2001; 72: 1251-1255.

63. Neer RM, Arnaud CD, Zanchetta JR, et al. Effect of parathyroid hormone (1-34) on fractures and bone mineral density in postmenopausal women with osteoporosis. $N$ Engl $J$ Med 2001; 344: 1434-1441.

64. Watts NB, Harris ST, Genant HK. Treatment of painful osteoporotic vertebral fractures with percutaneous vertebroplasty or kyphoplasty. Osteoporos Int 2001; 12: 429-437.

65. Lubetkin EI, Lipson DA, Palevsky HI, et al. GI complications after orthotopic lung transplantation. Am J Gastroenterol 1996; 91: 2382-2390.

66. Berkowitz N, Schulman LL, McGregor C, Markowitz D. 
Gastroparesis after lung transplantation. Potential role in postoperative respiratory complications. Chest 1995; 108: 1602-1607.

67. Palmer SM, Miralles AP, Howell DN, Brazer SR, Tapson VF, Davis RD. Gastroesophageal reflux as a reversible cause of allograft dysfunction after lung transplantation. Chest 2000; 118: 1214-1217.

68. Lau CL, Palmer SM, Howell DN, et al. Laparoscopic antireflux surgery in the lung transplant population. Surg Endosc 2002; 16: 1674-1678.

69. Goldstein LS, Haug MT 3rd, Perl J 2nd, et al. Central nervous system complications after lung transplantation. J Heart Lung Transplant 1998; 17: 185-191.

70. Thyagarajan GK, Cobanoglu A, Johnston W. FK506induced fulminant leukoencephalopathy after single-lung transplantation. Ann Thorac Surg 1997; 64: 1461-1464.

71. Nakamura M, Fuchinoue S, Sato S, et al. Clinical and radiological features of two cases of tacrolimus-related posterior leukoencephalopathy in living related liver transplantation. Transplant Proc 1998; 30: 1477-1478.

72. Lanzino G, Cloft H, Hemstreet MK, West K, Alston S, Ishitani M. Reversible posterior leukoencephalopathy following organ transplantation. Description of two cases. Clin Neurol Neurosurg 1997; 99: 222-226.

73. Drachman BM, DeNofrio D, Acker MA, Galetta S, Loh E. Cortical blindness secondary to cyclosporine after orthotopic heart transplantation: a case report and review of the literature. J Heart Lung Transplant 1996; 15: 1158-1164.

74. de Groen PC, Aksamit AJ, Rakela J, Forbes GS, Krom RA. Central nervous system toxicity after liver transplantation. The role of cyclosporine and cholesterol. N Engl J Med 1987; 317: 861-866.

75. Nussbaum ES, Maxwell RE, Bitterman PB, Hertz MI, Bula W, Latchaw RE. Cyclosporine A toxicity presenting with acute cerebellar edema and brainstem compression. Case report. J Neurosurg 1995; 82: 1068-1070.

76. Stang MR, Hinderliter AL, Gott KK, Paradowski LJ, Aris RM. Atrial anastomotic thrombus causes neurologic deficits in a lung transplant recipient. Transplantation 1996; 62: 693-695.

77. Lichtenstein GR, Yang YX, Nunes FA, et al. Fatal hyperammonemia after orthotopic lung transplantation. Ann Intern Med 2000; 132: 283-287.

78. Tuchman M, Lichtenstein GR, Rajagopal BS, et al. Hepatic glutamine synthetase deficiency in fatal hyperammonemia after lung transplantation. Ann Intern Med 1997; 127: 446449.

79. Berry GT, Bridges ND, Nathanson KL, et al. Successful use of alternate waste nitrogen agents and hemodialysis in a patient with hyperammonemic coma after heart-lung transplantation. Arch Neurol 1999; 56: 481-484.

80. Ishani A, Erturk S, Hertz MI, Matas AJ, Savik K, Rosenberg ME. Predictors of renal function following lung or heart-lung transplantation. Kidney Int 2002; 61: 22282234.

81. Pattison JM, Petersen J, Kuo P, Valantine V, Robbins RC,
Theodore $\mathbf{J}$. The incidence of renal failure in one hundred consecutive heart-lung transplant recipients. Am J Kidney Dis 1995; 26: 643-648.

82. Campistol JM, Sacks SH. Mechanisms of nephrotoxicity. Transplantation 2000; 69: S5-S10.

83. Camara NO, Matos AC, Rodrigues DA, Pereira AB Pacheco-Silva A. Early detection of heart transplant patients with increased risk of ciclosporin nephrotoxicity. Lancet 2001; 357: 856-857.

84. Ladefoged SD, Andersen CB. Calcium channel blockers in kidney transplantation. Clin Transplant 1994; 8: 128-133.

85. Klein IH, Abrahams A, van Ede T, Hene RJ, Koomans HA, Ligtenberg G. Different effects of tacrolimus and cyclosporine on renal hemodynamics and blood pressure in healthy subjects. Transplantation 2002; 73: 732-736.

86. Israni A, Brozena S, Pankewycz O, Grossman R, Bloom R. Conversion to tacrolimus for the treatment of cyclosporineassociated nephrotoxicity in heart transplant recipients. Am J Kidney Dis 2002; 39: E16.

87. Hernandez-Herrera G, Castillo DD, Perez R, Lopez-Rubio $\mathrm{F}$, Aljama P. Tacrolimus rescue therapy for cyclosporineinduced nephrotoxicity. Transpl Int 1998; 11: Suppl. 1, S104 S106.

88. Friemann S, Feuring E, Padberg W, Ernst W. Improvement of nephrotoxicity, hypertension, and lipid metabolism after conversion of kidney transplant recipients from cyclosporine to tacrolimus. Transplant Proc 1998; 30: 1240-1242.

89. Snell GI, Levvey BJ, Chin W, et al. Sirolimus allows renal recovery in lung and heart transplant recipients with chronic renal impairment. J Heart Lung Transplant 2002; 21: 540546

90. Young BA, Marsh CL, Alpers CE, Davis CL. Cyclosporineassociated thrombotic microangiopathy/hemolytic uremic syndrome following kidney and kidney-pancreas transplantation. Am J Kidney Dis 1996; 28: 561-571.

91. Galli FC, Damon LE, Tomlanovich SJ, Keith F, Chatterjee K, DeMarco T. Cyclosporine-induced hemolytic uremic syndrome in a heart transplant recipient. $J$ Heart Lung Transplant 1993; 12: 440-444.

92. Myers JN, Shabshab SF, Burton NA, Nathan SD. Successful use of cyclosporine in a lung transplant recipient with tacrolimus-associated hemolytic uremic syndrome. $J$ Heart Lung Transplant 1999; 18: 1024-1026.

93. Walder B, Ricou B, Suter PM. Tacrolimus (FK 506)-induced hemolytic uremic syndrome after heart transplantation. J Heart Lung Transplant 1998; 17: 1004-1006.

94. Neild GH, Ivory K, Williams DG. Glomerular thrombosis and cortical infarction in cyclosporin-treated rabbits with acute serum sickness. Br J Exp Pathol 1984; 65: 133-144.

95. Butkus DE, Herrera GA, Raju SS. Successful renal transplantation after cyclosporine-associated hemolyticuremic syndrome following bilateral lung transplantation. Transplantation 1992; 54: 159-162.

96. Singh N, Gayowski T, Marino IR. Hemolytic uremic syndrome in solid-organ transplant recipients. Transpl Int 1996; 9: 68-75. 